\title{
Using electronic health records for clinical trials: Where do we stand and where can we go?
}

\author{
Kimberly A. Mc Cord MSc, Lars G. Hemkens MD MPH
}

Cite as: CMAJ 2019 February 4;191:E128-33. doi: 10.1503/cmaj.180841

See related article at www.cmajopen.ca/lookup/doi/10.9778/cmajo.20180096

I

ncreasing interest in use of routinely collected data for research has been paralleled by a rising interest in using electronic health record (EHR) data for health research, as such records have become more widely used in clinical practice. ${ }^{1}$ In Canada, adoption of EHRs has been patchy and hampered by fragmentation of the health care system, variable funding streams, and different priorities of individual provinces and territories. ${ }^{2}$ However, both the government, by supporting the Canada Health Infoway, and nongovernmental organizations, such as the Canadian Medical Association and Canadian Pharmacists Association, have identified national adoption and interoperability of EHRs as a priority. ${ }^{2}$

A national EHR infrastructure could revolutionize health research in Canada and worldwide if EHRs were used to facilitate large-scale randomized studies. Traditional randomized controlled trials (RCTs) are often costly, and frequently use strict inclusion and exclusion criteria and standardized settings, which may mean that study findings are not generalizable to all populations. ${ }^{3}$ However, randomization within EHRs could allow largescale pragmatic RCTs to be conducted within the routine care setting, offering almost perfect generalizability of their findings. ${ }^{1,4-7}$ Such an approach would transform the evaluation of health care interventions, allowing continuous learning from series of systematic evaluations of variations of health care procedures and policies, with aggregated and shared information continuously fed back into the original systems (the "learning health care system"), and allowing agile improvements in clinical care, service delivery and the health system. ${ }^{8}$

We outline the potential benefits and difficulties of conducting RCTs using EHRs, drawing on a systematic evaluation of existing trials. $^{7}$

\section{How are EHRs currently used in clinical research?}

We recently evaluated systematically the current use of EHRs for RCTs (Box 1). ${ }^{7}$

We distinguish 2 general types of EHR-based RCTs: conventional trials, in which patients are recruited or outcomes are collected via EHRs (EHR-supported trials), and EHR-evaluating trials

\section{KEY POINTS}

- Randomized controlled trials (RCTs) using electronic health records (EHRs) can be directly integrated in routine care and allow large-scale and pragmatic trials with almost perfect generalizability.

- Currently, EHRs are mostly used in RCTs as part of the intervention, for example, when decision-support systems are evaluated as modifications or additions within the EHR infrastructure.

- Increasingly, EHR infrastructure is leveraged for patient recruitment or outcome assessment to support trials that assess conventional interventions.

- Randomized controlled trials within EHRs may address the limitations of traditional RCTs by increasing generalizability, reducing costs and time, expanding the research fields and allowing a democratization of research agendas.

- Important challenges include infrastructure costs, interoperability, standardization and quality of data, and ethical, privacy and data-security considerations.

that depend on the EHRs to actually deliver the intervention. Although most RCTs currently evaluate the EHR technology itself (e.g., by testing if adding an alert to a prescription software would reduce the rates of prescription mistakes), some RCTs have successfully used EHRs as supportive tools to facilitate recruitment and assessment of outcomes while testing an intervention not related to the EHR technology itself (e.g., by assessing rates of cardiovascular events after a particular complex intervention ${ }^{9}$.

Most EHR trials are currently performed in North America, where the uptake of EHRs has been promoted heavily by many stakeholders, and large health management organizations (e.g., Kaiser Permanente in the United States) have been developing their EHR infrastructures for several years. ${ }^{10}$

\section{How can an RCT be conducted within an EHR?}

Trials evaluating EHRs frequently aim to improve care by directing physicians through interventions involving clinical decision-support 
and computerized physician order entry systems (definitions in Table 1). This is done by identifying at-risk patients and flagging them, by monitoring the ordering system and submitting alerts in case of discrepancies, by displaying guidelines and advice

\section{Box 1: The use of electronic health records in} randomized controlled trials published since $2000^{7}$

- $91 \%$ of RCTs using EHRs evaluated the merits of EHR-based interventions, such as alerts or other clinical decision-support systems.

- There are some examples of RCTs supported by using EHRs for patient recruitment and outcome assessment alone.

- Most originated from North America (the United States and Canada).

- Most trials were published recently (median publication year 2012).

- Trials supported by EHRs cost between US\$44 and US\$2000 per patient.

Note: $\mathrm{EHR}=$ electronic health record, RCT = randomized controlled trial through the EHRs, or by providing auditing and feedback of health care performance. Thus, these trials mostly evaluate system-level as opposed to patient-level interventions; the latter can be assessed, for example, by leveraging personal health records, telehealth or electronic patient-reported outcomes (Table 1). All of these interventions may require additional software development and hardware that may, in turn, increase logistical difficulties and costs, particularly in telehealth trials.

Trials supported by EHRs, on the other hand, can be conducted in widespread EHR networks, such as the Veterans Affairs health system. ${ }^{13}$ Using EHRs for recruitment, typically with a retrospective procedure of simple backward querying of the EHR database, can help to obtain a list of possibly eligible patients. ${ }^{4}$ Some trials promote concurrent recruitment by sending an electronic message to the clinician or an automatically generated letter to the patient directly, ${ }^{14}$ and others use a more sophisticated data-mining approach to scrutinize the pharmacy EMR to flag particular patients, such as those with poorly controlled asthma ${ }^{15}$ (Boxes 2 and 3 ).

Table 1: Types and applications of electronic health records, and definitions

\section{EHR type and application}

Definition*

Type

Electronic health record (EHR)

Electronic medical record (EMR)

Application

Clinical decision-support system (CDSS)

Computerized physician order entry (CPOE) system

Personal health record (PHR)

Telehealth

Electronic patientreported outcomes (ePROs)
EHRs are electronic platforms that contain health-related data collected during medical care in practices, clinics and other medical settings from various sources, connected to form a network of patient clinical data. EHRs can also incorporate software that allow straightforward physician ordering practice (CPOEs), even including safety features, or that guide physicians through clinical decision-making with up-to-date guidelines (CDSS).

EMRs are routinely collected data sources that contain standard medical and clinical data gathered during medical care in an individual location of a practice, clinic or other medical setting. When the data are shared among different locations and units, it becomes a network and is considered an EHR (i.e., an electronic chart system in a primary care practice that cannot be accessed by any other entity is an EMR, whereas a hospital system in which laboratory data, affiliated clinic charts, etc., are all accessed under 1 platform is an EHR).

A CDSS is an application that supports health providers in performing health care by mining data of an EHR or EMR, and providing guideline-specific recommendations. These systems can often identify errors or missing data, and display alerts or messages through the EHRs.

CPOE systems are electronic ordering technologies in which physician orders can be entered and processed in a computerized way, often mimicking the workflow found in clinical settings. CPOE systems can be more advanced and identify ordering mistakes, display preferred treatments by individual patient EHR query, or even set up blocks with medication-interaction orders.

PHRs are electronic platforms (often online interfaces such as websites) that securely store patient's health information and allow patients to engage actively in their own health. Often, they can add information to a PHR, can exchange it with health providers, see test results, make appointments or receive educational information. We consider PHRs to be only those platforms that are tethered to an EHR, where information can be exchanged in both directions (otherwise, if patients are simply adding data but not viewing any of their data, we consider it an ePRO).

Telehealth is the use of telecommunication technologies (telemonitoring) to improve the provision of care. This allows for care to be provided at a distance and therefore to maintain clinical contact with patients at home without requiring the same amount of resources to be dispensed. Examples of telehealth are blood glucose-monitoring machines tethered to an EHR that integrate blood glucose levels (taken by the patient at home) into the EHR automatically (and can send an alert in the EHR interface to the clinician if the values are out of a predefined range and action must be taken), and, increasingly, mobile health data collected by wearable devices.

ePROs are health-related data recorded by the patient themselves in electronic form, often through a website or application. Whereas ePROs have often been used in clinical trials, we also consider ePROs to be any data that have been collected by the patients themselves and tethered to an EHR or PHR. An example would be a patient pain diary, in which a pain score and information are entered daily on a website or via a smartphone application, and these data are added to an EHR, which the clinician can monitor and consult during a visit. 
Box 2: Examples of randomized controlled trials based on electronic health records

\section{The Salford Lung Study ${ }^{16}$}

- GlaxoSmithKline sponsored a pre-approval trial involving 2802 patients conducted in the United Kingdom between 2014 and 2016.

- Patients with asthma or COPD were recruited in GP practices and asked if they would agree to be randomly assigned to receive a proprietary combination inhaler for asthma and COPD or usual care.

- Most outcome data were routinely collected from EHRs over 12 months.

- Overall, >80 GP sites and > 130 community pharmacies participated.

\section{Bereznicki 2008 ${ }^{15}$}

- 1551 patients were included between 2006 and 2007 to explore the effect of a data-mining application of medication records to improve asthma management.

- Pharmacy EHRs queried for patients nonadherent to asthma medication.

- Pharmacists collaborated with clinicians to improve medication adherence.

- 35 pharmacies participated.

Note: $\mathrm{COPD}=$ chronic obstructive pulmonary disease, $\mathrm{EHR}=$ electronic health record, $\mathrm{GP}=$ general practitioner.

\section{How can RCTs conducted within EHRs help to address the limitations of traditional RCTs?}

\section{Costs}

Randomized controlled trials are often expensive to conduct, but this is not because of their nature or study design. It is possible to conduct trials at low cost with high impact on patient care using smart designs and framing clear and simple research questions. ${ }^{6,19}$ The high burden of tasks involved in the development and maintenance of data collection is a main driver of trial costs, and setting up a specific data-collection infrastructure can be difficult in many medical settings. Innovative use of EHRs can reduce the costs associated with data collection in trials. We found a trend of substantial cost savings by using fully automated EHR-driven data. ${ }^{7}$ For example, the per-patient costs reported in a trial using fully automated EHR infrastructure was US\$44,,$^{15}$ compared with US\$2000 per participant in a less wellautomated trial. ${ }^{20}$ We provide more information on costs, including more reasons for such cost differences, elsewhere. ${ }^{7}$

\section{Time}

Even when funds are not an issue, just the decrease in logistical difficulties themselves, particularly in large RCTs, could be worth extracting routinely collected EHR data. Trials could be set up earlier and results obtained much faster. Ideally, results could be collected in real time, and first results could possibly be obtained even days after the implementation of changes. For example, the impact of certain control measures in infectious disease outbreaks could be directly assessed by using information centrally collected by EHRs.
Box 3: Examples of randomized controlled trials from Canada evaluating electronic health records

Tamblyn 2015

- The study was performed in the Quebec region and involved 81 primary care physicians and 4447 patients.

- Physicians received access to an asthma decision-support system, which identified patients with poorly controlled asthma and displayed evidence-based advice on management.

- After 3 to 33 months, the patient's use of asthma medication was monitored in the EHR.

Price $2017^{18}$

- The study was performed in British Columbia and involved 28 physicians and 23976 older patients.

- The study assessed the development of 40 prescribing rules in the EHR, aiming to reduce potentially inappropriate prescriptions.

- After 16 weeks, the rates of potentially inappropriate prescriptions were assessed, as well as physicians' experiences (mixed-methods approach).

Note: $\mathrm{EHR}=$ electronic health record

\section{Recruitment}

One of the largest advantages of using EHRs for clinical trials may be their facilitation of patient recruitment and of outcome assessment. Randomization can occur directly from the EHR during a patient's visit, minimizing any disruption of the flow of clinical care ${ }^{21}$ (Figure 1). Recruiting patients through the EHR allows prescreening for eligibility before approaching any potential participant, thus reducing effort required to enrol an appropriate patient sample. Rapid consecutive enrolment could favour recruitment through automatic screening and selection of participants within the EHR database ${ }^{4,21}$ (Figure 1), which could boost and speed up recruitment in trials requiring large samples. Poor recruitment is the most frequent reason for trials to be discontinued before reaching the required sample size, and overestimating the prevalence of eligible patients is the most frequently reported reason for recruitment failure; $;^{22,23}$ such trials waste many resources. Using EHRs to predict recruitment rates and as a recruiting framework is an innovation that may lead to enormous cost savings in research.

\section{Outcomes}

The ability to assess outcomes without having to measure or collect them in a dedicated data-collection system is another appealing advantage of using EHR data for RCTs. A frequently raised argument against clinical trials is that it would require too much time and too many patients to measure patient-relevant outcomes such as clinical events or death. However, outcomes such as stroke, myocardial infarction, hospital admissions or serious adverse effects are typically recorded in EHRs, and therefore the data are readily available. Evaluation of outcomes that are most relevant to decision-making in clinical care may also be encouraged when EHRs are used for large pragmatic trials. Trials that are more mechanistic in design (i.e., those aiming to better understand biological pathways and treatment mechanisms) could also 
benefit from using EHRs that include real-time information from wireless devices and monitoring systems, such as body weight, temperature, blood pressure or other vital parameters.

\section{Expanding the scope of research and democratizing research agendas}

Another advantage of using EHRs for prospective clinical research is that they offer a wide palette of options to answer research questions. For example, patient involvement may be expanded, evaluated and optimized by using personal health records (i.e., records that allow patients to receive important health information, to access their clinical data and to monitor progression of their health). ${ }^{24}$ It is now possible to connect telemedicine equipment, such as blood glucose-monitoring devices, via wireless data transmission to EHR systems, sending clinical parameters directly to the patient charts and allowing for remote monitoring of the patient clinical status. ${ }^{25}$ This information could be used for novel care models, which can be directly evaluated and optimized, to achieve system learning from innovation and evaluation. Furthermore, the ranges of interventions that can be assessed through EHRs are unprecedented. Electronic health records allow the add-on of interventions or software directly in the workflow of the clinical team, enabling clinicians to become the research team. Electronic health records open the doors to point-of-care trials, ${ }^{21,26}$ or trials embedded in clinical care, in which patients are automatically assessed for eligibility, included and randomly assigned directly at the clinician's side. Trials based on EHRs also lend themselves to the evaluation of nondrug treatments and interventions for which there is little evidence. For example, in the areas of speech therapy or physical therapy, patients could be assigned to different therapy regimens versus standard regimens, and evaluation of effectiveness and even cost-effectiveness can be conducted using outcome data from the EHR.

\section{What are the limitations of carrying out RCTs within EHRs?}

\section{Infrastructure costs}

Initial investments associated with implementing an EHR infrastructure that can facilitate research may be substantial. ${ }^{27}$ Although research stakeholders may save resources in the longer term, health care institutions may balk at the high cost of setting up and maintaining the infrastructure.

\section{Interoperability}

The technological potential of EHRs has developed more quickly than health care systems have been able to adapt and implement them, which has created an often fractured and unequal distribution of technologies across different jurisdictions, health care

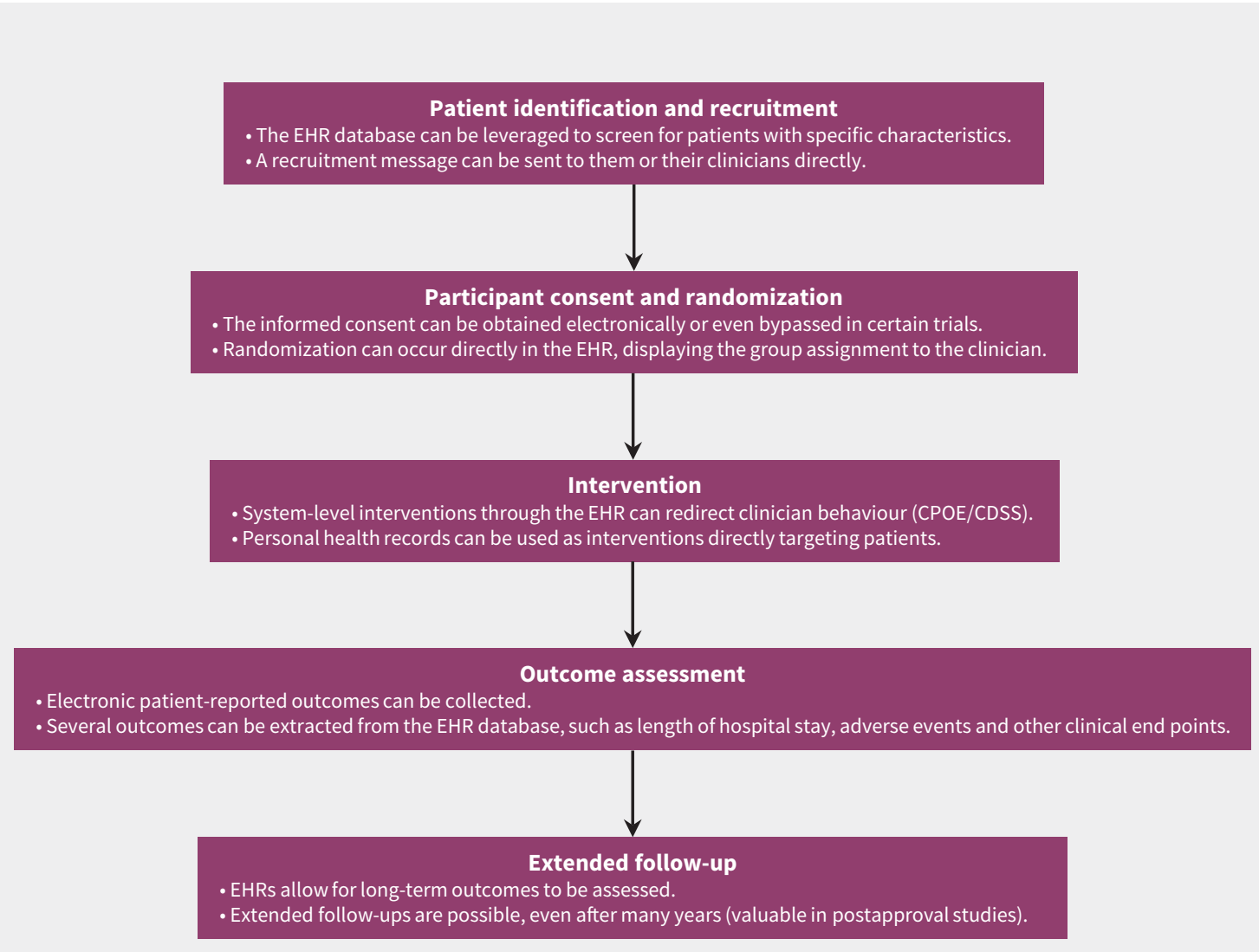

Figure 1: Practical applications of EHRs for randomized trials. Note: CDSS = clinical decision support system, CPOE $=$ computerized physician order entry, EHR = electronic health record. 
systems or facilities. A frequent limitation is the lack of interoperability of the EHR systems. ${ }^{28}$ Although 2 hospitals may well be routinely collecting useful clinical data using an EHR, it may be difficult to link 2 different systems to facilitate data collection and analysis in multicentre and international trials. With increasing uptake of EHRs, greater efforts will be needed to increase and promote their interoperability. Developers of EHRs and researchers would also need to come together and converge their interest. ${ }^{28}$ Nonetheless, even with perfect interoperability, EHRs do not often contain the complete medical history and health picture of patients, and trials using EHRs might require either a combination of data sources or a supplementation with active data collection. ${ }^{29}$

\section{Standardization and data quality}

Probably the largest limitation of using EHR data for research is that collection of data that is not primarily intended for research purposes naturally leads to problems of poor standardization both in the type of data collected and in the quality of data collection. ${ }^{30}$ When clinical staff enter information in electronic charts they often use free-text boxes; these data are typically not easily linked to electronic case report forms or trial databases. Text recognition, advanced algorithms and machine learning could help with data extraction, and artificial intelligence may help to streamline information. ${ }^{31}$ However, the completeness and granularity of the data may be compromised. ${ }^{32}$ Sometimes, clinical notes and discharge letters include context-related and "between-the-lines" information, which may be difficult to capture automatically. Because data collection for EHRs will always be oriented toward the individual patient, its format and intervals might never match those expected by clinical trials protocol; this requires that trialists give careful thought to their research question and potential adaptation of the design of their study. ${ }^{29}$

Different EHR systems, different staff members and different settings will probably all influence the detail and accuracy of data available for study. It is important that this is carefully considered and evaluated during the trial setup. Ideally, the accuracy of each data item, possible biases and misclassification risks are well described and quantified. Health care providers may need to be educated to understand the importance of proper documentation for the patient's well-being, particularly for important patient-relevant clinical outcomes. As the clinical workforce becomes more accustomed to using EHRs, it is reasonable to anticipate that the quality of EHR data available to researchers may improve. A continuous exchange between researchers and information technology specialists will likely be required to align the data format needs of researchers with the technical data-processing options and possibilities. In any case, the need for accurate data in randomized trials using EHRs is lower than in nonrandomized studies, which must measure much more information on potential confounders to ensure sufficient statistical adjustment.

\section{Privacy, data security and ethical considerations}

Finally, there is a need to consider the complex and multifaceted ethical and privacy issues related to using EHR data for research. Different consent systems may be necessary, for example, consent that is given for the data use and sharing on one hand (e.g., as in a cancer registry), and for the implementation of interventions on the other hand. Obtaining informed consent for automated trials conducted within EHRs may be difficult. ${ }^{4}$ It remains to be discussed whether informed consent needs to be acquired when only variations of usual care are explored. ${ }^{7} \mathrm{~A}$ disconnect exists between modern trials using EHRs and structures created for traditional RCTs (e.g., requirements of institutional review boards); however, with more examples of such trials being performed, it is likely that processes will alter and align. Further research and constructive dialogues among all stakeholders on this issue are urgently needed.

Data security remains paramount. ${ }^{33,34}$ Health care organizations are often caught between the desire to share EHR data for research advancement and the risk of data breaches in doing so. Introduction of innovative technologies in health care networks, such as big data analytics (with accompanying loss of anonymization ${ }^{35}$ ) or blockchain databases (with a potential increase in data safety as well as immutable audit trail ${ }^{36}$ ), will influence discussions on privacy and confidentiality of patient data.

\section{Conclusion}

Electronic health records have enormous potential to increase and to change the capacity of clinical health research by facilitating RCTs in the real-world setting. Many RCTs have successfully evaluated within-EHR interventions, recruited patients or assessed clinical end points with minimal patient contact. Uptake of EHRs is likely to increase in all health care settings, which will increase the amount of data available for research. Nonetheless, various stakeholders will need to be involved in ensuring that EHR implementation suits both the clinical workflow and clinical research requirements. We need EHR systems that ensure interoperability, provide standardized and high-quality data, and carefully address privacy and data security issues.

\section{References}

1. Hemkens LG, Contopoulos-loannidis DG, Ioannidis JP. Routinely collected data and comparative effectiveness evidence: promises and limitations. CMAJ 2016;188:E158-64

2. Chang F, Gupta N. Progress in electronic medical record adoption in Canada. Can Fam Physician 2015;61:1076-84.

3. Bothwell LE, Greene JA, Podolsky SH, et al. Assessing the gold standard - lessons from the history of RCTs. N Engl J Med 2016;374:2175-81.

4. Mc Cord KA, Al-Shahi Salman R, Treweek S, et al. Routinely collected data for randomized trials: promises, barriers, and implications. Trials 2018;19:29.

5. Zuidgeest MGP, Goetz I, Groenwold RHH, et al.; GetReal Work Package 3. Series: Pragmatic trials and real world evidence: paper 1. Introduction. J Clin Epidemiol 2017;88:7-13.

6. Hemkens LG. How routinely collected data for randomized trials provide longterm randomized real-world evidence. JAMA Netw Open 2018;1:e186014. doi: 10.1001/jamanetworkopen.2018.6014.

7. Mc Cord KA, Ewald H, Ladanie A, et al.; RCD for RCTs initiative and the Making Randomized Trials More Affordable Group. Current use and cost of electronic health records for clinical trial research: a descriptive study. CMAJ Open 2019;7:E23-32.

8. Friedman $\mathrm{CP}$, Wong AK, Blumenthal $\mathrm{D}$. Achieving a nationwide learning health system. Sci Transl Med 2010;2:57cm29.

9. Stewart JC, Perkins AJ, Callahan CM. Effect of collaborative care for depression on risk of cardiovascular events: data from the IMPACT randomized controlled trial. Psychosom Med 2014;76:29-37.

10. Chen C, Garrido T, Chock D, et al. The Kaiser Permanente Electronic Health Record: transforming and streamlining modalities of care. Health Aff (Millwood) 2009;28:323-33. 
11. What is an electronic health record (EHR)? Washington (DC): Office of the National Coordinator for Health Information Technology, U.S. Department of Health and Human Services; reviewed 2018 Mar. 21. Available: www.healthit. gov/faq/what-electronic-health-record-ehr (accessed 2019 Jan. 7).

12. Electronic health records. Baltimore: Centers for Medicare \& Medicaid Services; modified 2012 Mar. 26.

13. Goodman K, Krueger J, Crowley J. The automatic clinical trial: leveraging the electronic medical record in multi-site cancer clinical trials. Curr Oncol Rep 2012; 14:502-8.

14. Bender BG, Cvietusa PJ, Goodrich GK, et al. Pragmatic trial of health care technologies to improve adherence to pediatric asthma treatment: a randomized clinical trial. JAMA Pediatr 2015;169:317-23.

15. Bereznicki BJ, Peterson GM, Jackson SL, et al. Data-mining of medication records to improve asthma management. Med J Aust 2008;189:21-5.

16. Woodcock A, Bakerly ND, New JP, et al. The Salford Lung Study protocol: a pragmatic, randomised phase III real-world effectiveness trial in asthma. BMC Pulm Med 2015;15:160.

17. Tamblyn R, Ernst $P$, Winslade $N$, et al. Evaluating the impact of an integrated computer-based decision support with person-centered analytics for the management of asthma in primary care: a randomized controlled trial. J Am Med Inform Assoc 2015;22:773-83.

18. Price M, Davies I, Rusk R, et al. Applying STOPP guidelines in primary care through electronic medical record decision support: randomized control trial highlighting the importance of data quality. JMIR Med Inform 2017;5:e15.

19. Speich B, von Niederhäusern B, Blum CA, et al.; MAking Randomized Trials Affordable (MARTA) Group. Retrospective assessment of resource use and costs in two investigator-initiated randomized trials exemplified a comprehensive cost item list. J Clin Epidemiol 2018;96:73-83.

20. Piazza G, Anderson FA, Ortel TL, et al. Randomized trial of physician alerts for thromboprophylaxis after discharge. Am J Med 2013;126:435-42.

21. Ramsberg J, Neovius M. Register or electronic health records enriched randomized pragmatic trials: The future of clinical effectiveness and costeffectiveness trials? Nordic Journal of Health Economics 2015;5:62-76.

22. Kasenda B, von Elm E, You J, et al. Prevalence, characteristics, and publication of discontinued randomized trials. JAMA 2014;311:1045-51.
23. Kasenda B, von Elm EB, You J, et al. Learning from failure - rationale and design for a study about discontinuation of randomized trials (Drosoph Inf ServCO study). BMC Med Res Methodol 2012;12:131.

24. Tang PC, Ash JS, Bates DW, et al. Personal health records: definitions, benefits, and strategies for overcoming barriers to adoption. J Am Med Inform Assoc 2006;13:121-6

25. Dinesen B, Nonnecke B, Lindeman D, et al. Personalized telehealth in the future: a global research agenda. J Med Internet Res 2016;18:e53.

26. van Staa TP, Dyson L, McCann G, et al. The opportunities and challenges of pragmatic point-of-care randomised trials using routinely collected electronic records: evaluations of two exemplar trials. Health Technol Assess 2014;18: 1-146.

27. Choi JS, Lee WB, Rhee P-L. Cost-benefit analysis of electronic medical record system at a tertiary care hospital. Healthc Inform Res 2013;19:205-14.

28. Reisman M. EHRs: the challenge of making electronic data usable and interoperable. $P \& T$ 2017;42:572-5.

29. Hersh WR, Weiner MG, Embi PJ, et al. Caveats for the use of operational electronic health record data in comparative effectiveness research. Med Care 2013;51(Suppl 3):S30-7.

30. Capurro D, Yetisgen M, van Eaton E, et al. Availability of structured and unstructured clinical data for comparative effectiveness research and quality improvement: a multisite assessment. EGEMS (Wash DC) 2014;2:1079.

31. Miller DD, Brown EW. Artificial intelligence in medical practice: The question to the answer? Am J Med 2018;131:129-33

32. Cimino JJ. Improving the electronic health record - Are clinicians getting what they wished for? JAMA 2013;309:991-2.

33. Kruse CS, Smith B, Vanderlinden $\mathrm{H}$, et al. Security techniques for the electronic health records. J Med Syst 2017;41:127.

34. Ozair FF, Jamshed N, Sharma A, et al. Ethical issues in electronic health records: a general overview. Perspect Clin Res 2015;6:73-6.

35. Lasko TA, Vinterbo SA. Spectral anonymization of data. IEEE Trans Knowl Data Eng 2010;22:437-46.

36. Kuo TT, Kim HE, Ohno-Machado L. Blockchain distributed ledger technologies for biomedical and health care applications. J Am Med Inform Assoc 2017;24: 1211-20.
Competing interests: Kimberly Mc Cord and Lars Hemkens support the Routinely Collected Data for Randomized Controlled Trials Initiative (RCD for RCT initiative), which aims to explore the use of routinely collected data for clinical trials. They are members of The Making Randomized Trials Affordable (MARTA) Group. No other competing interests were declared.

This article has been peer reviewed.
Affiliations: Basel Institute for Clinical Epidemiology and Biostatistics, Department of Clinical Research, University Hospital Basel, University of Basel, Basel, Switzerland

Contributors: Both authors contributed to writing and revising the manuscript, approved the final version to be published and agreed to act as guarantors of the work,

Correspondence to: Lars Hemkens, lars.hemkens@usb.ch 